\title{
Naproxen sodium, diflunisal, and placebo in the treatment of chronic back pain
}

\author{
H. BERRY, ${ }^{1}$ BRENDA BLOOM, ${ }^{1}$ E. B. D. HAMILTON,$^{1}$ AND D. R. S WINSON ${ }^{2}$ \\ From the ${ }^{1}$ Department of Rheumatology and Rehabilitation, King's College Hospital, London SE5 9RS, and \\ the ${ }^{2}$ Department of Rheumatology, Wrightington Hospital, Wigan, Lancashire
}

SUMMARY Thirty-seven patients with chronic back pain were entered into a randomised, 3-way, double-blind, cross-over comparison of naproxen sodium $550 \mathrm{mg}$ twice daily, diflunisal $500 \mathrm{mg}$ twice daily, and placebo. Each treatment was given for 14 days after a preadmission wash-out week during which only paracetamol was allowed. Patients were assessed on admission and at the end of each treatment with respect to global pain, night pain, pain on movement, and pain on standing. Both visual analogue scales and simple descriptive scales were used to measure pain. Side effects were elicited by a nonleading question. Both methods of pain measurement gave similar results and were highly correlated. Naproxen sodium was superior to placebo in relieving global pain and, depending on the method of measurement, in relieving night pain and pain on movement. Diflunisal showed no significant differences from placebo. Side effects were similar on all 3 treatments. The final preference of the patients was significantly in favour of the active treatments.

Both naproxen sodium and diflunisal are analgesics with anti-inflammatory properties that are used primarily for their analgesic effect. Diflunisal has been heralded as the safer aspirin. In a controlled general-practitioner study ${ }^{1}$ it was reported that diflunisal had 3 advantages over aspirin in being more effective, more convenient (twice daily dosage), and less likely to cause gastric side effects in patients treated for acute pain. However, there has been some evidence ${ }^{2} 3$ to suggest that even this 'safe' drug may cause acute peptic ulcer. Naproxen sodium has been shown to produce significantly higher and earlier plasma levels of naproxen than an equivalent dose of the free acid, naproxen. ${ }^{4}$ It is therefore suited for use in acute conditions. Naproxen sodium has shown to be as effective as indomethacin in treating acute musculoskeletal disorders in general practice. ${ }^{5}$

The present study was set up to compare diflunisal and naproxen sodium in chronic back pain in outpatients attending a hospital clinic. A placebo was included to try to resolve the problem of comparing 2 similar drugs which may be equally effective or ineffective. The study was also designed to compare the 2 main methods of measuring pain, the visual analogue scale (VAS) and the simple descriptive 4-point scale (SDS).

Accepted for publication 20 February 1981

Correspondence to $\mathrm{Dr} \mathrm{H}$. Berry.

\section{Patients and methods}

Study design. The study was a double-blind, 3-way, cross-over comparison of naproxen sodium, diflunisal, and placebo in patients with chronic back pain of at least 3 months' duration. Patients satisfying the admission criteria were randomly allocated to one of the 6 possible orders of treatment. Any existing drug treatment for back pain was replaced by paracetamol for 7 days, after which the 3 test preparations were given in turn for 2 weeks.

Patients. Adult patients with chronic back pain due to spondylosis, degenerative spinal disease, sciatica, or pain of nonspecific cause were eligible for admission to the study. Patients with pain due to malignant disorders, infective diseases, or spondylolysthesis were excluded, as were patients with an alkaline phosphatase level outside normal limits and an ESR greater than $25 \mathrm{~mm}$ /hour.

Therapy. Naproxen sodium was given as two 275 $\mathrm{mg}$ capsules morning and evening $(1100 \mathrm{mg} /$ day $)$, diflunisal as two $250 \mathrm{mg}$ tablets morning and evening $(1000 \mathrm{mg} /$ day $)$, and placebo as a mixture of dummy naproxen sodium capsules and diflunisal tablets. A double-dummy method was used. No other nonsteroidal anti-inflammatory drugs were allowed during the course of the study, but patients were given a supply of paracetamol tablets for use as a rescue analgesic. Corsets, plaster, and physiotherapy were 
permitted so long as they were started before entry to the study and continued unchanged for the duration of the study.

Assessments. Patients were assessed at the end of the preadmission week on paracetamol and then at the end of each treatment period. Global pain, night pain, pain on movement, and pain on standing were assessed both on vertical $10 \mathrm{~cm}$ visual analogue scales and on conventional 4-point scales of none, mild, moderate, and severe. The patient's treatment preference was noted by comparing the first and second treatments, the second and third treatments, and at the end of the trial the overall preference. A record was kept of the use of the rescue analgesic in each period. Side effects were elicited by a nonleading question at the end of each treatment.

Statistical methods. Efficacy data from the SDS and VAS scales were analysed by a nonorthogonal analysis of variance.

\section{Results}

Thirty-seven patients, 24 women and 13 men, were entered into the study. Disease duration ranged from less than 1 year to 22 years, with a median of 3 years (see Table 1).

Changes in the efficacy measurements on treatments are shown in Fig. 1. The visual analogue scales and the conventional 4-point scales produced similar measurements of pain. Comparisons of the 2 scales by the Spearman coefficient of correlation shows statistically significant $(p<0 \cdot 05)$ correlations in all but 2 of the 40 comparisons made. However, as can be seen from Fig. 2, which shows the admission assessments, the overlap and spread of results were very large.

Both methods of pain measurement showed a statistically significant reduction of global pain on naproxen sodium, a significant increase of global pain on placebo, and no significant change on diflunisal. The difference between naproxen sodium and placebo was significant $(p<0.01)$ on both methods of pain measurement, but only the visual analogue scale showed a difference $(\mathrm{p}<0.05)$ between naproxen sodium and diflunisal.

A similar change was seen with night-pain results,

Table 1 Admission detail

\begin{tabular}{lc}
\hline Mean age & 55 years (range 32-79) \\
Males & 13 patients \\
Females & 24 patients \\
Median disease duration & 3 years (range $<1-22)$ \\
Lumbar/cervical spondylosis & 23 patients \\
Osteoporosis & 3 patients \\
Mild degenerative changes & 1 patient \\
Lordotic posture & 2 patients \\
Disc lesion & 4 patients \\
Nonspecific back pain & 4 patients \\
\hline
\end{tabular}

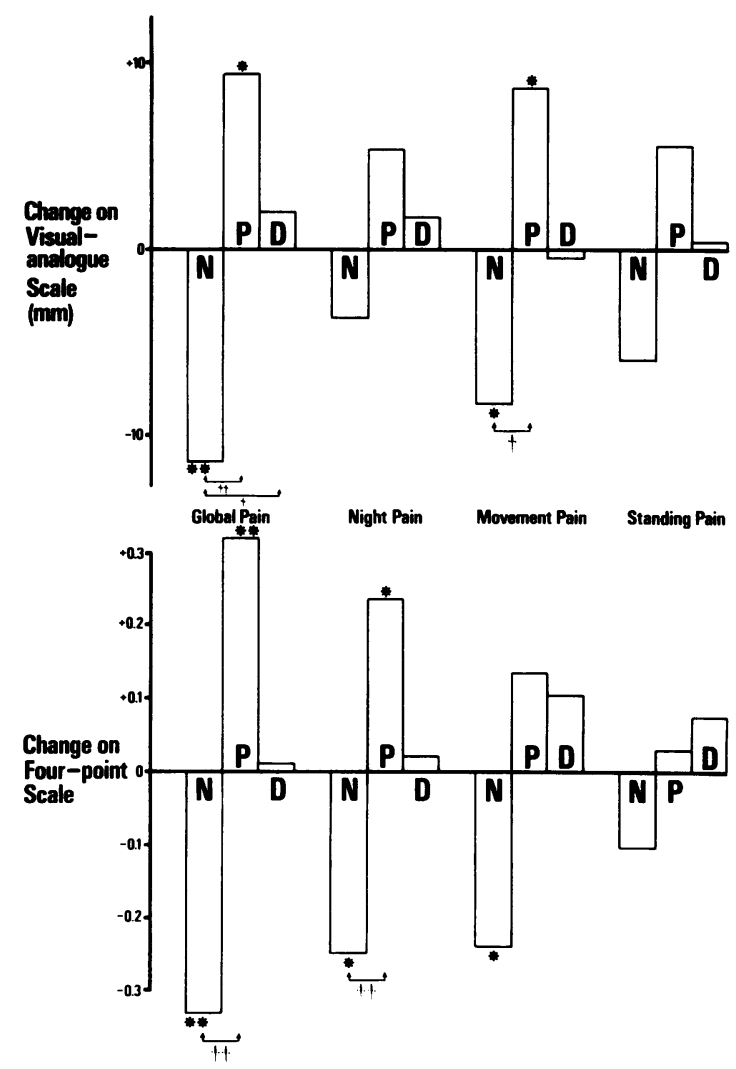

Fig. 1 Changes from admission in global pain, night pain, movement pain, and standing pain $(N=$ naproxen sodium, $P$ $=$ placebo,$D=$ diflunisal). Statistical significance of change from admission, ${ }^{*}=p<0.05,{ }^{* *}=p<0.01$ and comparison of changes on treatments $\dagger=p<0.05$, $\dagger+=p<0 \cdot 01$.

but here only the simple descriptive scale demonstrated significant differences between naproxen sodium and placebo, while the 2 active drugs were not significantly different.

Movement pain showed a reduction $(p<0 \cdot 05)$ on naproxen sodium regardless of which way it was measured, an increase on placebo (significant on the VAS), and no significant changes on diflunisal. The difference between naproxen sodium and placebo was significant on the VAS $(p<0 \cdot 05)$. Standing pain showed similar qualitative changes to global pain, night pain, and movement pain, but none was statistically significant.

Use of the rescue analgesic, paracetamol, showed no statistically significant differences between treatments, though there was a tendency for it to be low on naproxen sodium and high on placebo.

The final treatment preferences are shown in Table 

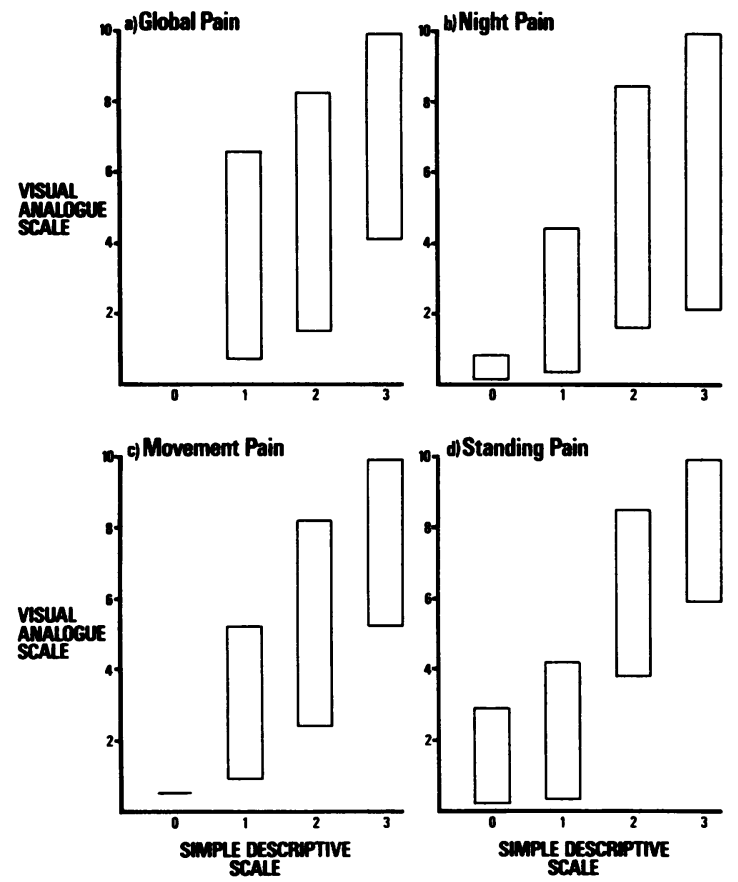

Fig. 2 Comparison of the 2 pain-rating scales at the admission visit.

2. There was a significant $(p=0.05$, chi-square) preference for both active treatments in comparison with placebo. There was no significant order effect.

During the naproxen sodium period 18 patients reported side effects, while on diflunisal 16 patients reported side effects; on placebo 18 patients reported side effects. The types of side effects reported are shown in Table 3 . The range of side effects was similar during all 3 treatment periods.

Reasons for discontinuation of drugs are summar-

Table 2 Final treatment preference

\begin{tabular}{lll}
\hline Naproxen sodium & Diflunisal & Placebo \\
\hline 14 & 13 & 4 \\
\hline
\end{tabular}

$p=0.05\left(\chi^{2}=5 \cdot 87 ; 2 D f\right)$

Table 3 Side effects

\begin{tabular}{lccc}
\hline & $\begin{array}{l}\text { Naproxen } \\
\text { sodium }\end{array}$ & Placebo & Diflunisal \\
\hline Gastrointestinal & 13 & 10 & 10 \\
CNS & 5 & 7 & 6 \\
Skin/nails & 2 & 3 & 2 \\
Urinary system & 0 & 0 & 2 \\
Total & 20 & 20 & 20 \\
Patients with side effects & 18 & 18 & 16 \\
\hline
\end{tabular}

Table 4 Trial withdrawals

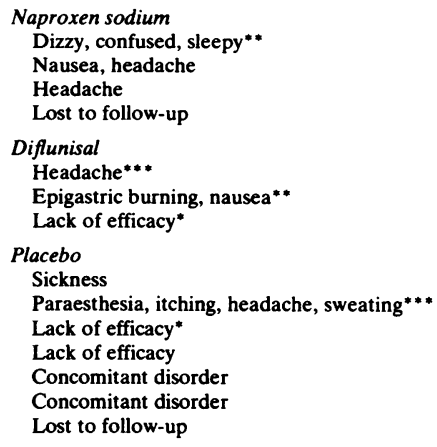

Same patients denoted by ${ }^{*}, * *, * * *$.

ised in Table 4. There were 14 drug discontinuations in 11 patients ( 3 patients stopped 2 of the 3 treatments) but only 4 patients failed to experience all three treatments.

\section{Discussion}

Regardless of the method used to measure pain, chronic back pain would appear to be a sensitive model on which to test analgesic drugs. In this study a clear difference was seen between the placebo and the test drugs and between the 2 test drugs. Naproxen sodium $550 \mathrm{mg}$ b.d. was superior to placebo in relieving global pain and showed some significant superiority over diflunisal $500 \mathrm{mg}$ b.d. Despite a 'wash-out week' on paracetamol prior to the admission assessment, patients still showed significant deterioration after 2 weeks on placebo. It is interesting that, although the clinical assessments clearly showed naproxen sodium to be superior to placebo and showed little difference between diflunisal and placebo the patients' preference placed naproxen sodium and diflunisal equally superior to placebo. However, as has already been shown, ${ }^{6}$ the patients' preference is a composite of many factors, of which efficacy and freedom from side effects may not be the most important. Both active drugs showed a similar side effect profile to the placebo. In the treatment of chronic back pain naproxen sodium must be considered superior to diflunisal.

Visual analogue scales have become increasingly popular since they were first introduced for use in rheumatology. ${ }^{7}$ This is because they employ a continuous scale and are highly sensitive to change. However, it is just this sensitivity which produces a great variability and hence inaccuracy in the results of such scales. The VAS, while being highly sensitive, can be highly inaccurate. Conversely the SDS, having a maximum range of only 4 or 5 units, is insensitive but 


\section{2}

owing to the greater ease with which patients grasp the concept is more accurate.

In this particular study the 2 scales appear to be measuring the same thing. Statistical tests of correlation show that the results of the 2 scales are highly correlated. However, the overlap of the results is great, showing a large difference in the calibration of the visual analogue scale by individual patients, which makes interpretation of results difficult. This confirms previously reported work. ${ }^{8}$ We feel that both methods should be used in studies of pain in order to gain as much information as possible about this important subjective phenomenon.

The present study shows no consistent advantage of either method of measurement. The 2 methods produced qualitatively similar results, and the differences in statistical significance may merely reflect the fact that more patients are required. Although there were variations in the statistical significance of the changes measured by the VAS and SDS in the study, the trends were the same, and naproxen sodium was consistently shown to be the better analgesic.

\section{References}

${ }^{1}$ Huskisson E C. Diflunisal-a new analgesic. Practitioner 1979; 223: 415-8.

- Talbot R, Rees H. Perforated duodenal ulcer on diflunisal (Dolobid). Br Med J 1978; ii: 1229.

${ }^{3}$ Mays A M. Bleeding massive gastric ulcer on diflunisal (Dolobid). Br Med J 1979; i: 888.

${ }^{4}$ Sevelius H, Runkel R, Segre E, Bloomfield S S. Bioavailability of naproxen sodium and its relationship to clinical analgesic effects. Br J Clin Pharmacol 1980; 10: 259-63.

${ }^{5}$ Backhouse C I, Engler C, English J R. Naproxen sodium and indomethacin in acute musculoskeletal disorders. Rheumatol Rehabil 1980; 19: 113-9.

- Berry H, Bloom B, Mace B E W, et al. Expectation and patient preference-does it matter? J $R$ Soc Med 1980; 73: 34-8.

${ }^{7}$ Berry H, Huskisson E C. Treatment of rheumatoid arthritis, a trial of meprothixol. Clin Trials $J$ 1972; 4: 13-5.

${ }^{8}$ Downie W W, Leathan P A, Rhind V M, Wright V, Branco J A, Anderson J A. Studies with pain relating scales. Ann Rheum Dis 1978; 37: 378-81. 